\title{
CAMBIO Y DESIGUALDAD \\ EN EL PROFESORADO \\ UNIVERSITARIO
}

\section{Amparo Almarcha Barbado, Benjamín González Rodríguez y Celia González Jorge}

Universidad Complutense de Madrid

\section{INTRODUCCION}

La variación operada en el mercado de trabajo y en general en la actividad de las mujeres fuera del campo tradicional del hogar se ha realizado, sin duda, a través de la transformación de modelos dentro del sistema educativo. El cambio en el mercado de trabajo, aunque vacilante todavía ${ }^{1}$, no hubiera podido producirse sin esta alteración en el ámbito educativo.

Dicho cambio está relacionado con aspectos de la modernización, con las características típicas de un régimen más democrático y con agentes sociales tales como las propias reivindicaciones feministas y movimientos de mujeres que actúan como dinamizadores de ese proceso. Puede decirse que el «área de la mujer» es una de las que más políticas sociales aglutina, probablemente porque sea más fácil, o menos conflictivo, intervenir en ella que tratar de erradicar

${ }^{1}$ La cifra de población activa femenina se situaba en 35,3 por 100 en el primer trimestre de 1994, y la de varones en 63,7 por 100. Téngase en cuenta que la proporción de ocupadas de la población potencialmente activa (16-64 años) no supera el 24,3 por 100. En cualquier caso, hay que señalar que mientras la cifra de mujeres ha ido subiendo en los últimos diez años (22,1 en 1983), la de varones ha descendido de un 58,7 por 100 en 1983 a 50,6 por 100 en 1994. Por otra parte, las tasas de paro masculino pasan de un 15,0 a un 20,7 por 100 en el mismo período. Sin embargo, entre las mujeres, el salto es el doble que entre los varones: 20,0 a 31,2 por 100 . Ministerio de Asuntos Sociales, Boletín Estadístico de Datos Básicos, núm. 16 (1994), pp. 52, 58 y 119. 
desigualdades socioeconómicas o de clase. De este modo, se ha extendido una mentalidad igualitaria entre los sexos y el sistema educativo aparece como un recinto aparentemente privilegiado para las mujeres. Todo ello origina una disparidad significativa entre mentalidades y realidades.

Así, el establecimiento de la coeducación, oponiéndose a la separación de sexos en la escuela (característica del régimen franquista), las campañas gubernamentales contra el sexismo en la escuela, las numerosas publicaciones sobre el lenguaje y prácticas escolares discriminatorias, y los manuales de texto no sexistas, entre otras, son realizaciones visibles en el panorama educativo español actual.

La expansión de una mentalidad y unas medidas sociopolíticas favorables a la no discriminación por razón de sexo, es un fenómeno internacional. Dentro del mundo educativo hay que destacar el gran logro que ha supuesto la consolidación de los «estudios sobre las mujeres» en el ámbito académico internacional. Los estudios interdisciplinares sobre las mujeres, es decir, sobre la división sexual vertebradora de nuestros sistemas sociales, han logrado el reconocimiento académico, de tal manera que su realización equivale al estudio de cualquier otra materia, en alguno de los países de la UE al igual que en EE.UU., pionero en este tipo de programas ${ }^{2}$.

En gran medida, a la influencia de este tipo de estudios se debe que se haya profundizado en la investigación de las diferencias sociosexuales, superando y modificando el análisis meramente estadístico, y se haya extendido a facetas antes inexploradas de esta realidad social.

\section{APUNTES SOBRE DESIGUALDAD Y EDUCACION}

Si observamos el sistema educativo de nuestro país podemos constatar una mayor dedicación por parte de las mujeres. Las mujeres apuestan por la educación: las tasas de escolarización de la población femenina tienden a ser más elevadas que las masculinas ${ }^{3}$, el rendimiento escolar de las mujeres es bastante

2 Sobre el desarrollo de este tipo de estudios, casi sin representación en nuestro país, véase M. A. GarCÍA DE LEÓN, "Cincuenta años de bibliografía sobre las mujeres en España (19401990). Análisis sociológico de la constitución de un nuevo campo científico, los "Estudios sobre las mujeres"», Anuario del Departamento de Historia III (1991).

${ }^{3}$ Proporción de alumnado por sexo y nivel educativo (Curso 88-89):

\begin{tabular}{|c|c|c|}
\hline & Mujeres & Varones \\
\hline 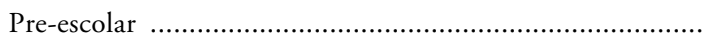 & 49,1 & 50,9 \\
\hline EGB & 48,3 & 51,7 \\
\hline BUP/COU & 54,8 & 45,2 \\
\hline FP & 43,4 & 56,6 \\
\hline 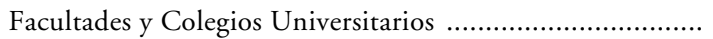 & 54,2 & 45,8 \\
\hline 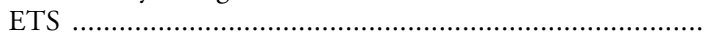 & 17,3 & 82,7 \\
\hline 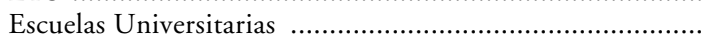 & 41,5 & 52,6 \\
\hline
\end{tabular}


superior como media tanto en la Educación General Básica ${ }^{4}$ como en Facultades y Escuelas Universitarias ${ }^{5}$. Dentro del mercado de trabajo, los seleccionadores de personal encuentran dificultades para poder rechazar a las candidatas femeninas, por su mayor preparación y adecuación a los perfiles de determinados puestos de trabajo ${ }^{6}$.

Estos y otros aspectos servirían para hacer el diagnóstico de la situación: las mujeres merecen pero no reciben en función de sus méritos, e incluso la desigualdad histórica en base a la variable sexo se radicaliza aún más cuando tenemos en cuenta la edad. Nuestra interpretación estaría relacionada, al menos, con un aspecto fundamental y que es consecuencia de un sistema educativo que hereda los valores tradicionales de una sociedad asimétrica ${ }^{7}$ y que, a su vez, intenta realizar el mito de la igualdad de oportunidades. Las mujeres pueden elegir entre alternativas aparentemente incompatibles: la familiar y la ocupacional, ambas igualmente oportunas, ventajosas y convenientes, pero, sin duda, algunas de las más preparadas para el mercado de trabajo prefieren cualquier sacrificio, incluso la doble jornada, antes que verse sólo como amas de casa.

${ }^{4}$ La proporción de alumnas que durante el curso 1988-89 obtuvo el título de graduado escolar era del 80,2 por 100 entre las mujeres, y del 74,4 por 100 entre los varones. Asimismo, la proporción de alumnos repetidores en el curso 1989-90 fue la siguiente:

\begin{tabular}{|c|c|c|c|}
\hline & & Mujeres & Varones \\
\hline 1. ${ }^{\circ} \mathrm{EGB}$ & $\ldots$ & 0,5 & 0,7 \\
\hline 5. ${ }^{\circ} \mathrm{EGB}$ & $\ldots$ & 3,8 & 5,1 \\
\hline 8. ${ }^{\circ} \mathrm{EGB}$ & (1) & 4,0 & 4,8 \\
\hline
\end{tabular}

FUENTE: Instituto de la Mujer, La mujer en cifras (Madrid, 1994, p. 38).

5 El porcentaje de alumnas que terminaron los estudios en Facultades Universitarias en el curso 1988-89 fue de 58,3 por $100,56,7$ por 100 en licenciaturas, 14,4 por 100 en Arquitectura e Ingeniería Técnica Superior, 15,5 por 100 en Ingenierías Técnicas y 73,8 por 100 en Diplomaturas (fuente: INE, Estadistica de la enseñanza superior. Curso 1989-90; los datos para el 88/89 son del Consejo de Universidades).

${ }^{6}$ La razón, según algunos de los jefes de personal de empresas como Iberia o El Corte Inglés, puede estar en que "las chicas necesitan demostrar lo que valen si quieren competir y se esfuerzan más que los chicos. El grado de motivación como factor psicológico es superior entre las mujeres, sobre todo las menores de veintiséis años, ya que no tienen que hacer la mili, no hay nada que las distraiga... ni siquiera el matrimonio». Sobre el rendimiento general de las mujeres, véase L. Bell y V. YOUNG «Impostoras, falsificadoras y estafadoras: Competencia y confianza en el empleo", en Not as far as you think: The realities of working woman (Lexington, Mass.: Lexington Books, 1986), cap. 3, pp. 25-51.

${ }^{7}$ La contradicción entre lo instrumental y lo expresivo, referida a los roles sexuales, aparece en la obra de T. PARSONS y R. BALES, Family, socialization and interaction process, Nueva York: Free Press, 1981. Véase también E. GIL CALVO, La mujer cuarteada: Utero, deseo y safo (Barcelona: Anagrama, 1991), especialmente capítulo III, pp. 62-85. 
El modelo actual de asimetría entre los géneros permite que las mujeres sigan trayectorias duales, lo cual produce una ambivalencia que a veces impide o dificulta el desarrollo de la propia estrategia vital. Las mujeres sometidas al doble vínculo se ven en la necesidad de estar eligiendo siempre entre la carrera familiar y la ocupacional, entre su rol expresivo y su rol instrumental.

Desde esta perspectiva son cuatro las condiciones que, a modo de hipótesis, se plantean para interpretar la desigualdad: a) una socialización diferencial de los sexos, a través del sistema escolar inicial; $b$ ) la necesaria relación entre clase social y sexo; $c$ ) un relativo fortalecimiento de las normas de igualdad a través de la escuela, y, por último, $d$ ) el prestigio social de los títulos superiores. Todo ello nos conduce a una nueva valoración y perspectiva del modelo femenino respecto a la educación y a las expectativas de cambio que este modelo puede generar y cuyo nivel de comprensión es social, necesitándose un enfoque análitico multifactorial.

\section{a) Socialización diferencial de los sexos, a través del sistema escolar inicial}

Varios estudios concluyen en el hecho de que la socialización de niñas y adolescentes implica valores de disciplina, orden y constancia en un nivel diferente y mayor que en el caso de los varones ${ }^{8}$. En éstos, los valores asociados a la llamada masculinidad tienen que ver con un interés por "ganarse pronto la vida», la autonomía y la salida al mercado de trabajo. De aquí su elección por estudios que posibiliten con mayor seguridad esa salida.

Por otra parte, los varones ven, y de hecho también tienen, más oportunidades que las mujeres en el mercado de trabajo. Como apuntábamos más arriba, la tasa de paro es diez puntos menor en el caso de los varones (21 por 100) respecto a las mujeres (31 por 100).

\section{b) Relación entre clase social y sexo}

Parece apuntarse como tendencia el hecho de un orden social más elevado de las mujeres estudiantes, fundamentalmente las universitarias, lo cual las aproximaría a un tipo de sistema cultural que aprecia en mayor medida los estudios. Esta podría ser una de las variables que explicaría la mejor y mayor adaptación de las mujeres a la norma y rutina escolar y a su mayor rendimiento ${ }^{9}$. Las mujeres estudiantes se centran en el estudio como primera aspiración y

${ }^{8}$ Véase la original contribución que sobre este tema ha realizado como tesis doctoral Lina Díaz, «Modelos de autopercepción social entre los alumnos de octavo de EGB».

9 Véase sobre este tema el estudio de Gloria Pérez Serrano, Origen social y rendimiento escolar (Madrid: CIS, 1981). 
a tiempo completo, mientras que en alguna medida los varones deciden trabajar, intentando compartir estudio y actividad en el mercado de trabajo ${ }^{10}$.

\section{c) Fortalecimiento de las normas de igualdad a través de la escuela}

Debido a la posición normativa de igualdad formal que proporciona la escuela, las jóvenes que deciden prepararse hacen un curriculum vitae en base a los estudios y eligen una forma más segura y/o cómoda que la competencia "abierta y despiadada» en el mercado de trabajo, cubierto por moldes sexistas y por los varones, a los cuales la sociedad impulsa a cumplir su rol privilegiado de participar en la sociedad ${ }^{11}$. Así, la «salida de la educación» constituye para las mujeres un privilegio único que algunos hombres desprecian a veces por su aparente facilidad.

\section{d) Prestigio social de los titulos superiores}

La necesidad de una mayor preparación en un sistema productivo inmerso en la tecnología se pone de manifiesto con la creación de nuevos puestos de trabajo que exigen titulación y especialización. La extensión de los títulos a más personas de la población, es decir, la universalización de la enseñanza, puede provocar una devaluación de los llamados diplomas y títulos escolares, lo cual permite una participación mayor de los más desfavorecidos por el modelo tradicional, entre ellos las mujeres. No obstante, la extensión de los títulos a la población femenina no conlleva que su consecución signifique la igualdad en el mercado de trabajo. Así, la tasa de paro de las universitarias en el primer trimestre de 1994 fue de 20,7 y, sin embargo, entre los universitarios fue de $11,9^{12}$.

\section{LA FEMINIZACION DE LA UNIVERSIDAD ESPAÑOLA}

Las mujeres han llegado a la Universidad española hace tan sólo unas décadas. La rapidez de la llegada, así como su inesperado y continuo crecimiento,

10 Según el «Informe de Juventud de 1988», los estudiantes varones trabajan y estudian en un 38 por 100 de los casos, frente a sólo el 25 por 100 de las mujeres.

11 Pero quizás, como indica Gil Calvo, irónicamente, no todo está tan claro: «A los chicos sólo se les ofrece una única carrera en la que tener que competir para poder triunfar, logrando el éxito en la lucha por la vida: la carrera ocupacional. Por ello, la amenazante sombra del fracaso acecha siempre inexorable, al carecerse de otra alternativa» (E. GIL CALVO, La mujer cuarteada..., cit., p. 81).

${ }_{12}$ Datos de la EPA, Ministerio de Asuntos Sociales, Boletín Estadístico de Datos Básicos, cit., p. 124 . 
han desconcertado a muchos de los observadores, políticos y científicos que, tanto desde fuera como desde el propio interior, han constatado la avalancha. Si en la década de 1950 a 1960 la participación era tan exigua, hoy la tendencia se ha invertido e incluso se ha producido una feminización de la Universidad española (tabla 1).

\section{TABLA 1}

Mujeres en el alumnado universitario

Años

1940-45 $1946-50 \quad 1951-55 \quad 1956-60 \quad 1960-68 \quad 1986-87 \quad 1989-90 \quad 1990-91$

$\begin{array}{lcccccccc}\text { Ambos sexos ...... } & 32.501 & 46.046 & 52.291 & 62.058 & 111.590 & 902.300 & 1.093 .086 & 1.137 .228 \\ \text { Mujeres ........... } & 5.032 & 6.275 & 8.660 & 11.932 & 34.677 & 452.400 & 554.490 & - \\ \text { \% de mujeres..... } & 13,0 & 13,5 & 15,0 & 19,0 & 30,0 & 50,1 & 50,7 & 52,5\end{array}$

FuENTES: Instituto de la Mujer, 1988, y Consejo de Universidades, Estadistica Universitaria 1991. Curso 1990/91. Datos facilitados por el Consejo de Universidades.

Los datos para el curso 1991/1992, referidos a la Universidad Complutense de Madrid, la más masificada de Europa, con un total de 130.141 alumnos, indican que un 60 por 100 de los nuevos ingresos son mujeres ${ }^{13}$. Lo más interesante de estos datos está precisamente en las asimetrías existentes entre el alumnado según ramas de estudio y sexo; si bien la enorme disparidad y supremacía del volumen total de estudiantes mujeres en las ramas de Humanidades y Ciencias Sociales con respecto a enseñanzas técnicas es un rasgo específico de la Universidad española que poco tiene que ver con el aspecto de desigualdad que estamos tratando (tabla 2 y gráfico 1 ).

Las ramas de Humanidades y Ciencias Sociales, junto con Ciencias de la Salud, muestran una mayor feminización. Ciencias Exactas y Naturales presentan un equilibrio relativo, puesto que Facultades como Ciencias Físicas prácticamente mantienen una proporción de alumnado femenino baja a lo largo de esos cinco cursos (27 ó 28 por 100), frente a Biológicas, que aparece como la más feminizada, seguida de Matemáticas y Químicas, que se mantienen en torno al 50 por 100 , y como contraste la rama de Ingeniería y Tecnología, en la que aparecen las mujeres como minoría. Si consideramos los datos relativos a las alumnas inscritas en primer curso de carrera, queda clara la tendencia a la subida en el grupo de las mujeres, tendencia que posiblemente no pueda mantenerse debido a la situación del mercado laboral, que parece proporcionar

${ }^{13}$ DAP, Información Estadistica de Alumnos. Curso 1991-92, p. 5. 


\section{TABLA 2}

Evolución del número de alumnos matriculados, clasificados por rama de enseñanza y sexo

\begin{tabular}{|c|c|c|c|c|c|c|c|c|c|c|c|c|}
\hline \multirow[b]{3}{*}{ Ramas de enseñanza } & \multicolumn{12}{|c|}{ Cursolalumnos } \\
\hline & \multicolumn{2}{|c|}{$1984-1985$} & \multicolumn{2}{|c|}{$1985-1986$} & \multicolumn{2}{|c|}{$1986-1987$} & \multicolumn{2}{|c|}{$1987-1988$} & \multicolumn{2}{|c|}{$1988-1989$} & \multicolumn{2}{|c|}{$1989-1990$} \\
\hline & $\begin{array}{c}\text { Ambos } \\
\text { sexos }\end{array}$ & $\begin{array}{c}\% \\
\text { mujeres }\end{array}$ & $\begin{array}{c}\text { Ambos } \\
\text { sexos }\end{array}$ & $\begin{array}{c}\% \\
\text { mujeres }\end{array}$ & $\begin{array}{l}\text { Ambos } \\
\text { sexos }\end{array}$ & $\begin{array}{c}\% \\
\text { mujeres }\end{array}$ & $\begin{array}{c}\text { Ambos } \\
\text { sexos }\end{array}$ & $\begin{array}{c}\% \\
\text { mujeres }\end{array}$ & $\begin{array}{c}\text { Ambos } \\
\text { sexos }\end{array}$ & $\begin{array}{c}\% \\
\text { mujeres }\end{array}$ & $\begin{array}{c}\text { Ambos } \\
\text { sexos }\end{array}$ & $\begin{array}{c}\% \\
\text { mujeres }\end{array}$ \\
\hline Todas las ramas & 788.168 & 48,4 & 854.104 & 49,5 & 902.284 & 50,1 & 969.412 & 50,5 & 1.027 .018 & 50,0 & 1.093 .086 & $6 \quad 50,7$ \\
\hline Humanidades y Ciencias Sociales & 496.101 & 55,3 & 547.129 & 56,5 & 581.207 & 57,2 & 628.903 & 57,9 & 667.460 & 57,4 & 707.251 & 158,1 \\
\hline Ciencias Exactas y Naturales ............. & 81.343 & 43,7 & 88.156 & 44,1 & 94.776 & 43,6 & 102.590 & 43,4 & 109.692 & 42,6 & 113.589 & $9 \quad 41,9$ \\
\hline 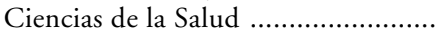 & 102.169 & 57,2 & 101.064 & 58,7 & 100.382 & 60,9 & 99.356 & 61,2 & 98.473 & 60,8 & 105.025 & $5 \quad 64,1$ \\
\hline Ingeniería y Tecnología ......... & 108.555 & 12,2 & 117.755 & 12,9 & 125.919 & 13,6 & 138.533 & 14,5 & 151.393 & 15,8 & 167.221 & $1 \quad 17,2$ \\
\hline
\end{tabular}




\section{GRAFICO 1}

\section{Distribución porcentual del alumnado por área de enseñanza y sexo}

(Curso 1989-90)

\section{MUJERES}

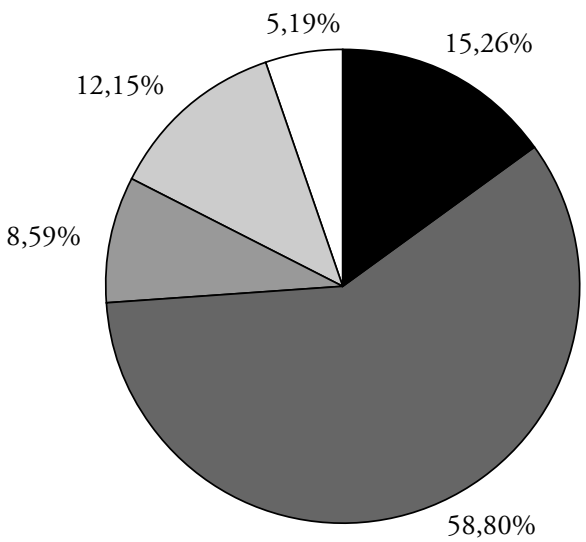

Humanidades

CC. Sociales y Jurídicas

CC. Exactas y Naturales

CC. de la Salud

Tecnológicas

\section{HOMBRES}

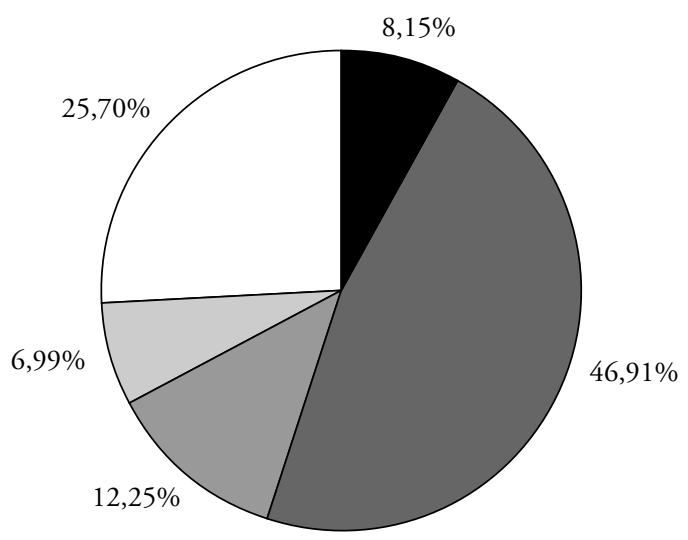

Humanidades

CC. Sociales y Jurídicas

CC. Exactas y Naturales

CC. de la Salud

Tecnológicas

FuENTE: Consejo de Universidades, Estadistica Universitaria 1991. 
cada vez menor cobijo a los licenciados universitarios ${ }^{14}$. Todo ello puede suponer que los varones vayan a las aulas en mayor proporción.

\section{LA ORIENTACION FEMENINA EN LA ELECCION DE CARRERA}

Hasta hace menos de una década, las decisiones universitarias parecían estar más de acuerdo con los gustos y opciones personales de vida, y podían explicar un poco mejor el condicionamiento previo de la variable género, es decir, de los procesos de socialización diferenciadores. En la actualidad esto puede estarse corrigiendo, si bien ésta es una hipótesis aventurada. La elección de opciones de carrera que debe realizar una buena parte del estudiantado impone, casi por la fuerza de la nota media de BUP y selectividad, la Facultad o Escuela que puede escoger el alumno. No obstante, hay carreras que son típicamente femeninas. La tabla 3 señala en detalle estas diferencias: un primer núcleo de Escuelas no Técnicas (Enfermería, Trabajo Social y Profesorado de EGB, entre otras).

Otro grupo está representado por las Escuelas Técnicas Superiores, donde se reduce la participación de las mujeres (10 por 100 en Ingeniería Naval e Ingeniería Industrial). Hay, por otra parte, una zona intermedia que se transforma de manera sorprendente y que aglutina a especialidades de rápido éxito en el mundo del trabajo: Medicina, Derecho y Económicas. Queda, finalmente, el reducto tradicional del alumnado femenino: Filosofía, Filología, Ciencias de la Educación, Geografía e Historia, Psicología y Farmacia. No obstante, según datos posteriores, se puede prever una subida importante de la proporción total de mujeres, tanto en las carreras de ciclo largo como en las de ciclo corto, sobre todo en estas últimas.

${ }_{14}$ Así, vemos que en las carreras de ciclo corto, las cursadas en Escuelas Universitarias, la tendencia es hacia un aumento de mujeres, excepto en Ingeniería y Tecnología, carreras en las que la tendencia se invierte. Por otra parte, en estas Facultades se recibe el 27 por 100 de mujeres en primer curso. Este dato tendría que estar relacionado con un mayor rendimiento académico femenino, tal como vimos anteriormente.

Proporción de mujeres matriculadas en primer curso (1990/91)

\begin{tabular}{|c|c|c|}
\hline & $\begin{array}{l}\text { Facultades } \\
\text { (ciclo largo) }\end{array}$ & $\begin{array}{c}\text { Escuelas Universitarias } \\
\text { (ciclo corto) }\end{array}$ \\
\hline 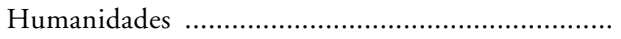 & 64,0 & 78,0 \\
\hline 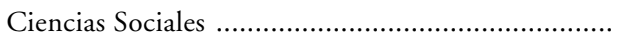 & 55,0 & 63,2 \\
\hline 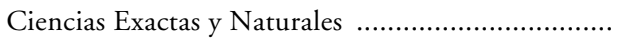 & 47,9 & 50,0 \\
\hline 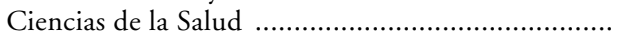 & 63,2 & 79,0 \\
\hline 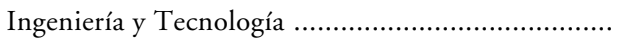 & 27,1 & 21,4 \\
\hline 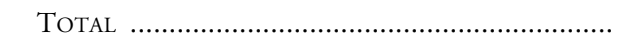 & 60,7 & 39,3 \\
\hline
\end{tabular}

FUENTE: Consejo de Universidades, 1994. 


\section{TABLA 3}

Evolución del número de alumnos matriculados, clasificados por titulación, enseñanza y sexo

\begin{tabular}{|c|c|c|c|c|c|c|}
\hline \multirow[b]{2}{*}{$\begin{array}{l}\text { Titulación } \\
\text { Enseñanzas }\end{array}$} & \multicolumn{2}{|c|}{$1985-86$} & \multirow{2}{*}{$\begin{array}{c}\% \\
\text { mujeres } \\
1985-86\end{array}$} & \multicolumn{2}{|c|}{$1989-90$} & \multirow{2}{*}{$\begin{array}{c}\% \\
\text { mujeres } \\
1989-90\end{array}$} \\
\hline & $\begin{array}{c}\text { Ambos } \\
\text { sexos }\end{array}$ & Mujeres & & $\begin{array}{c}\text { Ambos } \\
\text { sexos }\end{array}$ & Mujeres & \\
\hline Todas las enseñanzas & 854.104 & 422.440 & 49,5 & 1.093 .086 & 554.490 & 50,7 \\
\hline Licenciaturas & 576.896 & 304.770 & 52,8 & 701.679 & 386.648 & 55,1 \\
\hline Bellas Artes . & 8.321 & 4.815 & 57,9 & 10.628 & 6.196 & 58,3 \\
\hline CC. Biológicas & 26.751 & 14.632 & 54,7 & 24.953 & 14.097 & 56,5 \\
\hline CC. de la Educación & - & - & - & 13.682 & 10.974 & 80,2 \\
\hline CC. de la Información & 16.617 & 8.815 & 53,0 & 21.818 & 12.887 & 59,1 \\
\hline CC. del Mar . & - & - & - & 516 & 264 & 51,2 \\
\hline CC. Económicas y Empresariales ... & 72.307 & 25.782 & 35,7 & 126.297 & 54.196 & 42,9 \\
\hline 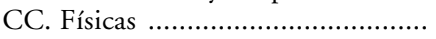 & 9.856 & 2.694 & 27,3 & 14.519 & 4.162 & 28,7 \\
\hline CC. Geológicas & 2.767 & 991 & 35,8 & 3.364 & 1.263 & 37,5 \\
\hline CC. 1 & 9.022 & 4.560 & 50,5 & 11.263 & 5.744 & 51,0 \\
\hline ogía ............... & 5.108 & 2.831 & 55,4 & 13.263 & 7.166 & 54,0 \\
\hline CC. $\mathrm{C}$ & 17.918 & 8.448 & 47,1 & 23.508 & 11.663 & 49,6 \\
\hline Derecho & 137.412 & 63.950 & 46,5 & 173.470 & 90.481 & 52,2 \\
\hline Derecho Canónico .......................... & 81 & 17 & 21,0 & 80 & 12 & 15,0 \\
\hline 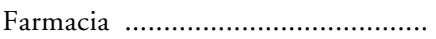 & 22.729 & 15.793 & 69,5 & 24.795 & 17.163 & 69,2 \\
\hline Filolog & 48.815 & 35.421 & 72,6 & 57.281 & 42.581 & 74,3 \\
\hline Filosofía & - & - & - & 5.844 & 3.075 & 52,6 \\
\hline Filosofía y CC. de la Educación ...... & 34.299 & 22.621 & 66,0 & 12.685 & 8.555 & 67,4 \\
\hline Filosofía y Letras & 6.665 & 4.411 & 66,2 & - & - & - \\
\hline Geografía e Historia & 54.695 & 33.019 & 60,4 & 49.753 & 29.282 & 58,9 \\
\hline Inform & 8.153 & 2.704 & 33,2 & 12.147 & 3.602 & 29,7 \\
\hline . & 47.678 & 24.120 & 50,6 & 40.426 & 23.145 & 57,3 \\
\hline ogía ................................... & - & - & - & 1.834 & 1.072 & 58,5 \\
\hline . & 33.984 & 24.096 & 70,9 & 46.522 & 33.323 & 71,6 \\
\hline . & 686 & 128 & 18,7 & 677 & 134 & 19,8 \\
\hline 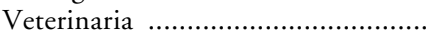 & 13.032 & 4.922 & 37,8 & 12.354 & 5.611 & 45,4 \\
\hline a e Ingenierias ... & 53.701 & 7.591 & 14,1 & 71.235 & 54 & 19,0 \\
\hline 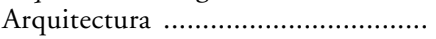 & 15.103 & 3.452 & 22,9 & 17.059 & 4.987 & 29,2 \\
\hline Ingenieros Aeronáuticos .................. & 1.727 & 113 & 6,5 & 1.559 & 197 & 12,6 \\
\hline I. Agrónomos . ....................................... & 4.407 & 1.004 & 22,8 & 5.976 & 1.757 & 29,4 \\
\hline I. de Caminos, Canales y Puertos ... & 4.517 & 366 & 8,1 & 6.182 & 824 & 13,3 \\
\hline (n........................ & 17.182 & 1.357 & 7,9 & 25.879 & 3.400 & 13,1 \\
\hline I. de Minas & 1.596 & 213 & 13,3 & 1.937 & 315 & 16,3 \\
\hline I. de Montes. & 965 & 185 & 19,2 & 992 & 261 & 26,3 \\
\hline I. Navales ..... & 608 & 50 & 8,2 & 778 & 125 & 16,1 \\
\hline 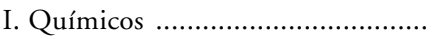 & 463 & 139 & 30,0 & 577 & 238 & 41,2 \\
\hline I. de Telecomunicación ................... & 5.945 & 516 & 8,7 & 8.925 & 1.267 & 14,2 \\
\hline Marina Civil (Náutica) .................... & - & - & - & 374 & 18 & 4,8 \\
\hline Primero de ETS .................................... & 1.188 & 196 & 16,5 & 997 & 165 & 16,5 \\
\hline
\end{tabular}




\section{TABLA 3 (continuación)}

Evolución del número de alumnos matriculados, clasificados por titulación, enseñanza y sexo

\begin{tabular}{|c|c|c|c|c|c|c|}
\hline \multirow[b]{2}{*}{$\begin{array}{l}\text { Titulación } \\
\text { Enseñanzas }\end{array}$} & \multicolumn{2}{|c|}{$1985-86$} & \multirow{2}{*}{$\begin{array}{c}\% \\
\text { mujeres } \\
1985-86\end{array}$} & \multicolumn{2}{|c|}{$1989-90$} & \multirow{2}{*}{$\begin{array}{c}\% \\
\text { mujeres } \\
1989-90\end{array}$} \\
\hline & $\begin{array}{c}\text { Ambos } \\
\text { sexos }\end{array}$ & Mujeres & & $\begin{array}{c}\text { Ambos } \\
\text { sexos }\end{array}$ & Mujeres & \\
\hline Arquitectura e Ingenierías Técnicas .. & 64.054 & 7.650 & 11,9 & 95.986 & 15.249 & 15,9 \\
\hline Arquitectura Técnica ...................... & 9.951 & 1.877 & 18,9 & 14.582 & 3.264 & 22,4 \\
\hline Ingeniería Técnica Aeronáutica ....... & 1.856 & 129 & 7,0 & 1.683 & 217 & 12,9 \\
\hline 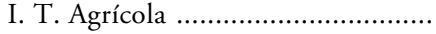 & 9.150 & 2.317 & 25,3 & 13.740 & 3.950 & 28,7 \\
\hline I. T. Forestal & 1.166 & 268 & 23,0 & 1.933 & 500 & 25,9 \\
\hline I. T. Industrial .. & 31.607 & 2.205 & 7,0 & 46.237 & 4.677 & 10,1 \\
\hline I. T. Minera & 1.695 & 113 & 6,7 & 2.257 & 258 & 11,4 \\
\hline I. T. Naval & 530 & 38 & 7,2 & 1.312 & 132 & 10,1 \\
\hline I. T. de Obras Públicas & 1.712 & 186 & 10,9 & 3.687 & 560 & 15,2 \\
\hline I. T. de Telecomunicación & 5.420 & 345 & 6,4 & 8.400 & 1.225 & 14,6 \\
\hline 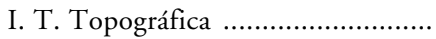 & 967 & 172 & 17,8 & 2.155 & 466 & 21,6 \\
\hline Diplomaturas ................................... & 159.453 & 102.429 & 64,2 & 224.186 & 139.039 & 62,0 \\
\hline Biblioteconomía y Documentación & 1.120 & 864 & 77,1 & 2.253 & 1.691 & 75,1 \\
\hline 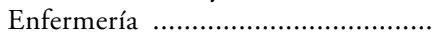 & 17.416 & 14.383 & 82,6 & 20.228 & 17.036 & 84,2 \\
\hline 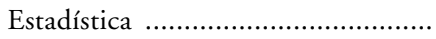 & 785 & 332 & 42,3 & 1.647 & 789 & 47,9 \\
\hline Estudios Empresariales .................... & 44.520 & 19.433 & 43,7 & 73.471 & 36.650 & 49,9 \\
\hline 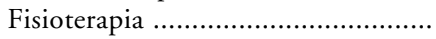 & 209 & 151 & 72,2 & 1.649 & 1.117 & 67,7 \\
\hline Graduados Sociales..$\ldots \ldots \ldots \ldots \ldots \ldots \ldots$ & - & - & - & 29.733 & 17.890 & 60,2 \\
\hline 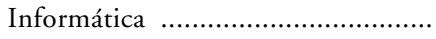 & 10.585 & 3.166 & 29,9 & 21.672 & 6.045 & 27,9 \\
\hline 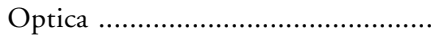 & 2.319 & 1.325 & 57,1 & 3.501 & 2.088 & 59,6 \\
\hline 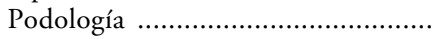 & - & - & - & 238 & 130 & 54,6 \\
\hline Profesorado de EGB . & 75.410 & 56.818 & 75,3 & 56.747 & 44.527 & 78,5 \\
\hline Trabajo Social & 5.848 & 5.041 & 86,2 & 10.960 & 9.398 & 85,7 \\
\hline Traductores e Intérpretes ................ & 1.241 & 916 & 73,8 & 2.087 & 1.678 & 80,4 \\
\hline
\end{tabular}

FUENTE: INE, Estadística de la Enseñanza universitaria 1985-86, y Consejo de Universidades, Estadística Universitaria 1991. 


\section{EL SIMBOLISMO DE LA CARRERA}

Para completar las diferencias que nos permiten explicar la desigualdad deberíamos estudiar la lógica del sistema de enseñanza, que es la del mercado de bienes simbólicos, como si el elegir la carrera fuera un bien de consumo más. Las mujeres, sin un horizonte profesional claro, ya que son recién llegadas a la enseñanza superior y a un hipotético mundo profesional, coinciden en su falta de orientación e información con los "otros estudiantes», y están de acuerdo con ellos también por la falta de una herencia profesional, la cual corresponde por lo general al varón (hermano o padre), mientras que a ellas, orientadas de antemano por un "destino femenino», se les deja más libertad de opción, libertad que puede entenderse también como una cierta indiferencia familiar hacia el tipo de estudios que elijan. De hecho, algunos padres prefieren tener "recogidas» a sus hijas en las aulas universitarias, independientemente del destino de sus carreras.

Los varones de clase media y media alta tienen un modelo profesional masculino, mientras que las mujeres, aparentemente más libres, con independencia de su clase social, no tienen un modelo profesional femenino claro. Para gran parte de ellas, no hay a quién parecerse. Por otra parte, algunas de estas futuras profesionales no tienen madres activas; en la mayoría de los casos, son "amas de casa" dedicadas a sus labores ${ }^{15}$. En todo caso, las mujeres no parecen rendirse, y siguen preparándose para el ejercicio de la actividad profesional, o bien por el simple estímulo de su propio desarrollo intelectual. Así, en el curso $1988 / 89$, un 38,2 por 100 de mujeres estaban matriculadas en cursos de doctorado y un 21,6 por 100 habían leído y aprobado sus tesis doctorales.

Del mismo modo que no hay referencia a un modelo profesional femenino específico en el contexto familiar, tampoco lo hay en el ámbito social, ya que se da una escasez de mujeres en bastantes campos profesionales.

Finalmente, los hombres evitan cursar carreras con un techo profesional limitado, como es el caso de las carreras de "letras», que conducen casi indefectiblemente a la docencia, mientras que las mujeres no las rechazan. Rehúyen, sin embargo, las que implican directamente una salida al mundo laboral en la esfera de lo privado; por ejemplo, las ingenierías superiores y, sobre todo y significativamente, las ingenierías técnicas. Parecen buscar la protección de un título superior o la posibilidad de acumular diplomas escolares a modo de remedio que suavice la discriminación que de hecho van a encontrar en el mercado de trabajo.

La creación de otros tipos de estudios, así como la aparición de nuevas carreras, parecen estar destinadas a los jóvenes más socializados en la competitividad y con capacidad para alejarse de especialidades o carreras saturadas.

15 En el caso de la Complutense, el 76,9 por 100 de las universitarias tiene madres no activas en el mercado de trabajo externo a las actividades domésticas. Universidad Complutense de Madrid, Información estadística de alumnos. Curso 1991-92 (Madrid, 1993), p. 42. 
Por otra parte, las mujeres jóvenes sienten la tensión y las contradicciones que ha proporcionado esta estructura tan consolidada de desigualdad. Así, el porcentaje de mujeres graduadas en el curso 1988/89 fue del 53,8 por 100. La distribución por carreras de estas mujeres graduadas ha ido incrementándose en la última década, tal como se aprecia en los datos siguientes. Esto podría apuntar la idea de un mayor rendimiento escolar femenino ${ }^{16}$.

Porcentaje de mujeres graduadas, por especialidades y curso

\begin{tabular}{|c|c|c|c|c|c|c|}
\hline & \multicolumn{3}{|c|}{ Escuelas Universitarias (ciclo corto) } & \multicolumn{3}{|c|}{ Facultades (ciclo largo) } \\
\hline & $1981 / 82$ & $1985 / 86$ & 1988/89 & $1981 / 82$ & $1985 / 86$ & 1988/89 \\
\hline Humanidades .......................... & . 85,5 & 55,6 & 79,4 & 62,9 & 64,0 & 66,6 \\
\hline Ciencias Sociales ..................... & . 69,0 & 70,9 & 72,3 & 41,2 & 44,0 & 53,9 \\
\hline Ciencias Exactas y Naturales. & 71,4 & 51,4 & 46,2 & 46,0 & 43,1 & 49,0 \\
\hline Ciencias de la Salud .................. & 75,8 & 79,9 & 84,6 & 45,0 & 51,7 & 54,7 \\
\hline Ingeniería y Tecnología ......... & 7,1 & 12,5 & 17,4 & 8,7 & 14,0 & 17,3 \\
\hline TOTAL . & . 61,8 & 62,7 & 65,5 & 46,1 & 49,3 & 53,8 \\
\hline
\end{tabular}

No obstante, las disparidades del porcentaje de graduadas, según carreras, permite asegurar la consolidación de títulos y profesiones «femeninas» en la actualidad, con un mercado más saturado que el de las ingenierías y tecnologías, que siguen reservadas preferentemente para los varones.

${ }^{16}$ Según los datos del INE para el curso 1987/1988, el porcentaje de alumnos que terminó sus estudios en el plazo oficial previsto, es decir, sin retraso académico, fue superior para las mujeres en todas las carreras:

\begin{tabular}{|c|c|c|c|}
\hline & Varones & Mujeres & Total \\
\hline Facultades y Colegios Universitarios .......................... & 38,7 & 46,8 & 18,9 \\
\hline ETS ..... & 18,9 & 23,4 & 21,2 \\
\hline 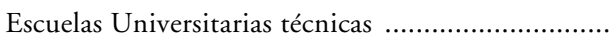 & 19,5 & 20,1 & 19,8 \\
\hline Escuelas Universitarias no técnicas ...................... & 34,6 & 60,2 & 47,4 \\
\hline
\end{tabular}




\section{EL PROFESORADO UNIVERSITARIO FEMENINO: LA BARRERA DE CRISTAL}

Existe una alta participación de las mujeres en los niveles de enseñanza no universitaria, reduciéndose esta presencia en los niveles de la enseñanza superior ${ }^{17}$.

Existe una participación diferencial según ramas de enseñanza, que tiende a asociar las letras con el campo en el cual las mujeres tienen mayor representatividad, frente al de las ciencias, que sería de ámbito masculino, pero lo cierto es que esta relación (tabla 4) no se produce de modo perfecto. Tan sólo el caso de la rama de ingeniería y tecnología presenta esa participación diferencial. El porcentaje de profesorado femenino no ha oscilado mucho en las diferentes ramas de enseñanza, ya que tan sólo ha crecido desde un 23,5 por 100 en el curso $1981 / 82$ a un 29,1 por 100 en el curso 1989/90.

Si analizamos estos mismos datos teniendo en cuenta el centro y el tipo de enseñanza (tabla 5) comprobaremos que un mayor flujo de alumnado femenino, típico de las carreras de Letras, no siempre supone un porcentaje más alto de profesoras. Incluso la falta de participación femenina es más notoria en Letras. Sólo Farmacia parece conocer un acoplamiento, cercano al 50 por 100, entre el alumnado y el profesorado femenino. Ciencias Políticas y Sociología, con un 54 por 100 de alumnas, está en torno a la media de la participación de alumnas en las Facultades, teniendo, sin embargo, menos profesoras $(22,8$ por 100) que Geológicas (31,1 por 100).

Para algunos países europeos los datos son similares a los de España. Así, en el caso de Holanda, había un 26,5 por 100 de profesoras en el curso 1986/87. En Francia, la cifra era del 27 por 100 en 1988/89, y en Inglaterra alcanzaba el 10 por 100 el profesorado femenino de educación superior en $1982^{18}$.

${ }^{17}$ La proporción femenina, según niveles de enseñanza, es la siguiente:

\begin{tabular}{|c|c|}
\hline & $\%$ de profesoras \\
\hline Pre-escolar & 83,9 \\
\hline 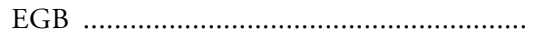 & 61,0 \\
\hline BUP y COU & 53,7 \\
\hline 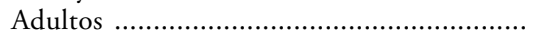 & 45,8 \\
\hline $\mathrm{FP} \quad \ldots \ldots \ldots \ldots \ldots \ldots \ldots \ldots \ldots \ldots \ldots \ldots \ldots$ & 40,7 \\
\hline Otras enseñanzas de segundo grado ............. & 44,3 \\
\hline 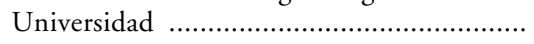 & 29,1 \\
\hline
\end{tabular}

FUENTE: INE, Estadistica de la enseñanza en España. Niveles de Pre-escolar, EGB, Enseñanzas Medias y Enseñanza Universitaria (Madrid, 1993).

${ }_{18}$ F. Massit-Folleá y F. Epinatte, L'Europe des Universités. L'enseignement supérieur en mutation (París: La Documentation Française, 1992). 


\section{TABLA 4}

Evolución del profesorado femenino, clasificado por rama de enseñanza (en porcentajes)

\begin{tabular}{|c|c|c|c|c|c|}
\hline Rama de enseñanza & Curso 1981-82 & Curso 1982-83 & Curso 1983-84 & Curso 1984-85 & Curso 1985-86 \\
\hline Todas las ramas & 23,51 & 24,18 & 24,87 & 24,87 & 26,77 \\
\hline Humanidades y Ciencias Sociales ....................... & 30,54 & 30,57 & 30,90 & 31,19 & 33,26 \\
\hline 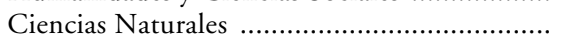 & 27,55 & 27,42 & 28,57 & 26,40 & 28,77 \\
\hline 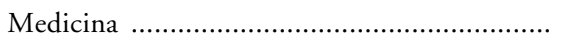 & 25,13 & 26,20 & 26,41 & 26,57 & 28,65 \\
\hline 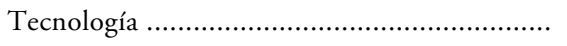 & 7,40 & 8,75 & 10,13 & 10,54 & 11,39 \\
\hline Rama de enseñanza & Curso 1986-87 & Curso 1987-88 & Curso 1988-89 & Curso 1989-90 & \\
\hline 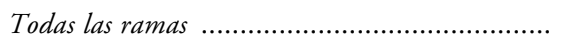 & 28,07 & 28,45 & 29,17 & 29,05 & \\
\hline 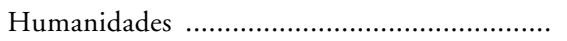 & 36,16 & 35,93 & 37,69 & 37,51 & \\
\hline 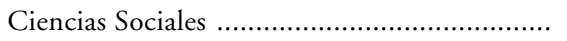 & 34,13 & 33,53 & 33,97 & 33,72 & \\
\hline Ciencias Exactas y Naturales ............................... & 28,83 & 29,25 & 29,55 & 29,12 & \\
\hline 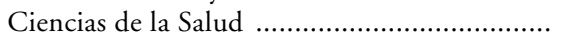 & 29,94 & 30,49 & 31,02 & 31,22 & \\
\hline 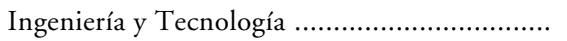 & 11,84 & 12,72 & 13,77 & 13,81 & \\
\hline
\end{tabular}

FuEntE: Consejo de Universidades, Ministerio de Educación y Ciencia, Anuario de Estadística Universitaria (Madrid, 1989 y 1992). 


\section{TABLA 5}

Evolución del número de profesores, clasificados por titulación, enseñanza y sexo

\begin{tabular}{|c|c|c|c|c|c|c|}
\hline \multirow[b]{2}{*}{$\begin{array}{l}\text { Titulación } \\
\text { Enseñanzas }\end{array}$} & \multicolumn{2}{|c|}{$1985-86$} & \multirow{2}{*}{$\begin{array}{c}\% \\
\text { mujeres } \\
1985-86\end{array}$} & \multicolumn{2}{|c|}{$1989-90$} & \multirow{2}{*}{$\begin{array}{c}\% \\
\text { mujeres } \\
1989-90\end{array}$} \\
\hline & $\begin{array}{c}\text { Ambos } \\
\text { sexos }\end{array}$ & Mujeres & & $\begin{array}{c}\text { Ambos } \\
\text { sexos }\end{array}$ & Mujeres & \\
\hline Todas las enseñanzas ....... & 45.390 & 12.189 & 26,9 & 56.953 & 16.546 & 29,1 \\
\hline Licenciaturas . & 27.224 & 7.365 & 27,1 & 34.736 & 9.938 & 28,6 \\
\hline Bellas Artes ............ & 432 & 109 & 25,2 & 747 & 186 & 24,9 \\
\hline 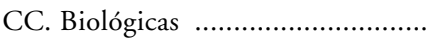 & 2.967 & 765 & 25,8 & 3.483 & 977 & 28,1 \\
\hline CC. de la Educación ....................... & 987 & 401 & 40,6 & 1.213 & 479 & 39,5 \\
\hline CC. de la Información & 517 & 100 & 19,3 & 704 & 146 & 20,7 \\
\hline CC. del Mar ...... & - & - & - & 45 & 10 & 22,2 \\
\hline CC. Económicas y Empresariales ... & 2.488 & 471 & 18,9 & 3.645 & 869 & 23,8 \\
\hline CC. Físicas …..................................... & 414 & 79 & 19,1 & 493 & 96 & 19,5 \\
\hline CC. Geológicas & 199 & 56 & 28,1 & 257 & 80 & 31,1 \\
\hline CC. Matemáticas .......................... & 404 & 87 & 21,5 & 421 & 82 & 19,5 \\
\hline CC. Políticas y Sociología ............... & 338 & 72 & 21,3 & 421 & 96 & 22,8 \\
\hline CC. Químicas ................................ & 1.075 & 337 & 31,3 & 1.068 & 361 & 33,8 \\
\hline (n) & 2.695 & 573 & 21,3 & 3.744 & 932 & 24,9 \\
\hline Derecho Canónico .......................... & 48 & - & - & 39 & - & - \\
\hline 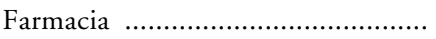 & 1.249 & 610 & 48,8 & 1.429 & 671 & 47,0 \\
\hline (n..................... & 1.098 & 486 & 44,3 & 1.432 & 675 & 47,1 \\
\hline Filosofía y CC. de la Educación ...... & 1.045 & 289 & 27,7 & 1.738 & 553 & 31,8 \\
\hline 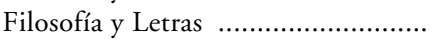 & 2.707 & 897 & 33,1 & 2.695 & 965 & 35,8 \\
\hline Geografía e Historia ...................... & 1.058 & 420 & 39,7 & 1.278 & 500 & 39,1 \\
\hline 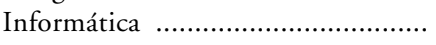 & 340 & 94 & 27,6 & 539 & 134 & 24,9 \\
\hline 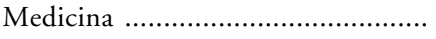 & 5.893 & 1.167 & 19,8 & 7.186 & 1.430 & 19,9 \\
\hline (n) & - & - & - & 316 & 83 & 26,3 \\
\hline 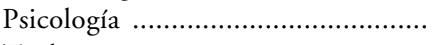 & 412 & 146 & 35,4 & 710 & 283 & 39,9 \\
\hline 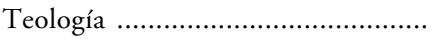 & 177 & - & - & 170 & 3 & 1,8 \\
\hline 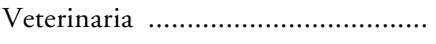 & 681 & 206 & 30,2 & 963 & 327 & 34,0 \\
\hline Arquitectura e Ingenierias ................ & 5.102 & 504 & 9,9 & 5.855 & 734 & 12,5 \\
\hline Arquitectura ................................. & 1.200 & 128 & 10,7 & 1.407 & 183 & 13,0 \\
\hline Ingenieros Aeronáuticos .................... & 144 & 6 & 4,2 & 142 & 7 & 4,9 \\
\hline I. Agrónomos . .................................... & 560 & 126 & 22,5 & 615 & 133 & 21,6 \\
\hline I. de Caminos, Canales y Puertos ... & 560 & 26 & 4,6 & 615 & 46 & 7,5 \\
\hline 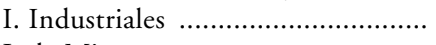 & 1.752 & 144 & 8,2 & 1.970 & 243 & 12,3 \\
\hline 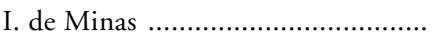 & 245 & 17 & 6,9 & 258 & 36 & 14,0 \\
\hline I. de Montes ..................................... & 128 & 17 & 13,3 & 123 & 11 & 8,9 \\
\hline 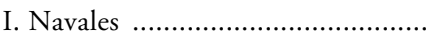 & 96 & 3 & 3,1 & 94 & 7 & 7,4 \\
\hline 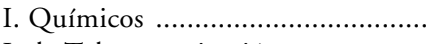 & 68 & 7 & 10,3 & 72 & 5 & 6,9 \\
\hline I. de Telecomunicación .................. & 349 & 30 & 8,6 & 481 & 55 & 11,4 \\
\hline Marina Civil (Náutica) ................... & - & - & - & 78 & 8 & 10,3 \\
\hline
\end{tabular}




\section{TABLA 5 (continuación)} Evolución del número de profesores, clasificados por titulación,
enseñanza y sexo

\begin{tabular}{|c|c|c|c|c|c|c|}
\hline \multirow[b]{2}{*}{$\begin{array}{l}\text { Titulación } \\
\text { Enseñanzas }\end{array}$} & \multicolumn{2}{|c|}{$1985-86$} & \multirow{2}{*}{$\begin{array}{c}\% \\
\text { mujeres } \\
1985-86\end{array}$} & \multicolumn{2}{|c|}{$1989-90$} & \multirow{2}{*}{$\begin{array}{c}\% \\
\text { mujeres } \\
1989-90\end{array}$} \\
\hline & $\begin{array}{c}\text { Ambos } \\
\text { sexos }\end{array}$ & Mujeres & & $\begin{array}{c}\text { Ambos } \\
\text { sexos }\end{array}$ & Mujeres & \\
\hline Arquitectura e Ingenierías Técnicas .. & 4.230 & 463 & 10,9 & 5.222 & 796 & 15,2 \\
\hline Arquitectura Técnica & 556 & 61 & 11,0 & 434 & 59 & 13,6 \\
\hline Ingeniería Técnica Aeronáutica ...... & 108 & 13 & 12,0 & 87 & 14 & 16,1 \\
\hline 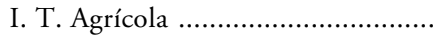 & 546 & 84 & 15,4 & 518 & 111 & 21,4 \\
\hline 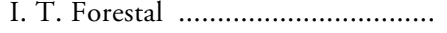 & 57 & 9 & 15,8 & 58 & 13 & 22,4 \\
\hline I. T. Industrial ..... & 1.870 & 208 & 11,1 & 1.517 & 217 & 14,3 \\
\hline I. T. Minera & 166 & 10 & 6,0 & 188 & 21 & 11,2 \\
\hline 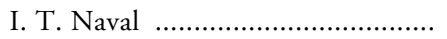 & 72 & 6 & 8,3 & 77 & 6 & 7,8 \\
\hline I. T. de Obras Públicas ................... & 164 & 9 & 5,5 & 179 & 9 & 5,0 \\
\hline I. T. de Telecomunicación & 207 & 16 & 7,7 & 289 & 25 & 8,7 \\
\hline I. T. Topográfica . & 52 & 11 & 21,2 & 40 & 11 & 27,5 \\
\hline 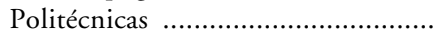 & 432 & 36 & 8,3 & 1.835 & 310 & 16,9 \\
\hline 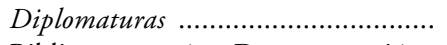 & 8.834 & 3.857 & 43,7 & 11.140 & 5.078 & 45,6 \\
\hline Biblioteconomía y Documentación & 31 & 17 & 54,8 & 82 & 42 & 51,2 \\
\hline Enfermería .................................. & 2.694 & 1.064 & 39,5 & 3.094 & 1.533 & 49,5 \\
\hline 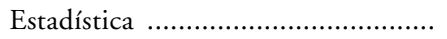 & 30 & 11 & 36,7 & 55 & 20 & 36,4 \\
\hline Estudios Empresariales .................... & 1.139 & 312 & 27,4 & 1.654 & 557 & 33,7 \\
\hline 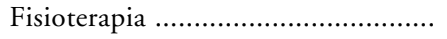 & 18 & 1 & 5,6 & 128 & 28 & 21,9 \\
\hline Graduados Sociales ......................... & - & - & - & 496 & 121 & 24,4 \\
\hline (n)...................... & 280 & 73 & 26,1 & 525 & 120 & 22,9 \\
\hline 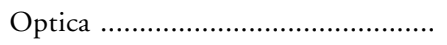 & 111 & 44 & 39,6 & 155 & 72 & 46,5 \\
\hline Podología & - & - & - & 18 & 6 & 33,3 \\
\hline Profesorado de EGB ....................... & 4.096 & 2.104 & 51,4 & 4.225 & 2.191 & 51,9 \\
\hline 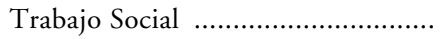 & 361 & 187 & 51,8 & 574 & 306 & 53,3 \\
\hline Traductores e Intérpretes ............... & 74 & 44 & 59,5 & 134 & 82 & 61,2 \\
\hline
\end{tabular}

FUENTE: INE, Estadística de la Enseñanza universitaria 1985-86, y Consejo de Universidades, Estadistica Universitaria 1991. 


\section{CATEGORIA ACADEMICA DE LAS DOCENTES}

A la Universidad han llegado las mujeres, como se ha señalado, pero, a pesar de la rapidez de su llegada, probablemente deban esperar más tiempo para ocupar los puestos docentes en pie de igualdad respecto a los varones. Hace una década, la participación de las mujeres era aún muy exigua; sólo nos diferencian de aquella etapa 7 puntos en el porcentaje, pero el signo estadístico positivo, a favor de las mujeres, no parece haber modificado lo que en aquel entonces observábamos: el elitismo de la Universidad. La Universidad de los años noventa, sigue siendo elitista, aunque se haya masificado. Las mujeres sólo han logrado "colarse» en los pequeños resquicios que han ido quedando, algunos de ellos despreciados por los varones, de los que se espera competitividad y mayor arrojo para salir a un mercado de trabajo más incómodo, aparentemente, que el de la enseñanza superior ${ }^{19}$. De aquel mítico 17,9 por 100 de mujeres profesoras del curso $1977-78$, se ha llegado a un 29,1 por 100 en el curso 1989-90. Si bien la cifra de participación de las mujeres está cercana a la media de población activa del mercado general de trabajo, lo cierto es que la distribución según la categoría académica y el sexo es sumamente reveladora de las posiciones de poder y control que siguen ejerciendo los varones en la organización y distribución de los puestos de trabajo dentro de la Universidad, así como en las formas de llegada y selección del nuevo profesorado (tabla 6).

La puesta en marcha de la LRU y las siguientes reformas de la enseñanza superior no parece que hayan servido para grandes cambios, como no sea el hecho de incrementar el funcionariado en la enseñanza superior. Si tenemos en cuenta el conjunto del profesorado, debemos concluir lo siguiente: 1) Se ha producido un estancamiento. La Universidad no ofrece perspectivas ni siquiera para los varones, ya que el cuerpo de catedráticos, que supone la máxima aspiración para muchos de los profesores de esta Universidad, presenta una tendencia a la baja entre el curso 85-86 y el 89-90. 2) La euforia reductora de tipos de categorías propuesta por las reformas de la LRU no tiene casi ningún significado. De algún modo, los encargados de curso han sido sustituidos por los asociados, cuyo perfil inicial consistía en conectar la Universidad con la sociedad, llevando a las aulas a profesionales prestigiosos que pudieran aportar la realidad práctica y mucho de sus profesiones. 3) A la vista de los datos señalados, pensamos que la Universidad no parece interesada en que algunos de sus profesores aprendan a enseñar si tenemos en cuenta que los ayudantes han pasado de un 22 por 100 en 1985/1986 a un 8,6 por 100 en 1989/1990.

19 A. Almarcha, Autoridad y privilegio en la Universidad española: Estudio sociológico del profesorado universitario (Madrid: CIS, 1982), pp. 119 y ss. En este estudio se toma en cuenta precisamente la escasísima participación de las mujeres en el ámbito de profesores numerarios, casi como un mal endémico. Refiriéndose a los catedráticos, la cifra utilizada era de un 2 por 100; los agregados, un 4 por 100, y los adjuntos, un 25 por 100. El estudio citado se refería a una muestra de nueve Facultades de diez distritos académicos, realizada en 1976 desde el IOP. F. Alvira y C. Collazos, El rol de profesor universitario (Madrid: INCIE, 1976) (mimeo). 


\section{TABLA 6}

Proporción del profesorado de Enseñanza Superior, por categoría académica y sexo

\begin{tabular}{|c|c|c|c|}
\hline \multirow[b]{2}{*}{ Categorias } & \multicolumn{3}{|c|}{ Curso $1985-1986$} \\
\hline & Varones & Mujeres & Total \\
\hline Catedráticos y Agregados ......................... & 17,6 & 4,1 & 13,9 \\
\hline Titulares & 34,9 & 32,6 & 34,3 \\
\hline Encargados de Curso ................................. & 17,8 & 16,2 & 17,4 \\
\hline 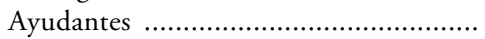 & 18,6 & 29,9 & 21,7 \\
\hline Maestros de Taller y Laboratorio ........... & 0,04 & 1,1 & 0,1 \\
\hline 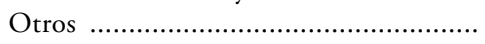 & 10,7 & 16,8 & 12,4 \\
\hline \multirow[t]{2}{*}{ 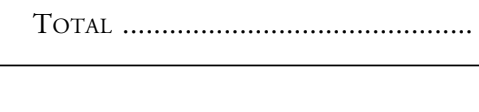 } & 100,0 & 100,0 & 100,0 \\
\hline & \multicolumn{3}{|c|}{ Curso 1989-1990 } \\
\hline Categorías & Varones & Mujeres & Total \\
\hline Catedráticos y Agregados .......................... & 15,5 & 4,4 & 12,5 \\
\hline 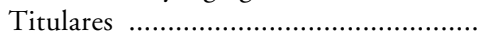 & 41,0 & 45,8 & 42,3 \\
\hline 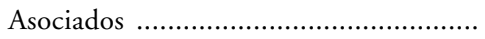 & 29,6 & 27,5 & 29,0 \\
\hline Ayudantes & 6,5 & 14,6 & 8,6 \\
\hline 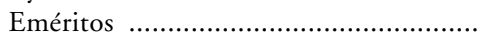 & 0,8 & 0,4 & 0,7 \\
\hline Maestros de Taller y Laboratorio ........... & 0,6 & 0,3 & 0,5 \\
\hline 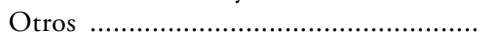 & 5,9 & 7,0 & 6,3 \\
\hline TOTAL & 100,0 & 100,0 & 100,0 \\
\hline
\end{tabular}

FUENTE: INE, Estadística de la Enseñanza Universitaria en España (Madrid, 1987 y 1991).

Por otra parte, con respecto a las mujeres, la situación es mucho más alarmante, por varias razones: 1) El sistema sigue siendo meritocrático y desigual, ya que la barrera de cristal no permite a las mujeres llegar más allá de la categoría de Titular, si no es a costa de un gran sacrificio familiar y personal. 2) La proporción de catedráticas, la élite del profesorado, sigue siendo la misma (4,4 por 100). 3) Sólo el 25 por 100 del total de asociados y un 16 por 100 del total de los eméritos son mujeres. Esta última categoría académica está propiciando el viejo sueño del Ministerio de «aprovechar» al máximo el profesorado y, además, a más bajo coste económico. 4) Se observa asimismo la desigualdad en las categorías menos consolidadas: más del doble de los ayudantes son mujeres $(14,6)$ con respecto a los varones $(6,5)$. 


\section{PODER Y PARTICIPACION ENTRE LOS PROFESORES UNIVERSITARIOS}

Pese a la situación minoritaria del profesorado femenino universitario, con una media de participación de un 29 por 100 en todas las enseñanzas (tabla 4), sobre todo si se la compara con el abundantísimo profesorado femenino del resto de los niveles educativos, se advierte, no obstante, una tendencia a incrementarse sensiblemente, por los factores ya apuntados. Ahora bien, ¿por qué la Universidad ha sido un ámbito que tradicionalmente ha atraído a los varones, conformando un universo masculinizado, cuando, por el contrario, éstos están poco motivados a trabajar en la enseñanza? En primer lugar, el trabajo en la Universidad ha sido, hasta hace poco tiempo, un trabajo ligero y compatible con otro trabajo principal al que añadía prestigio. Así, bastantes abogados y médicos famosos han estado, en muchas ocasiones, en esta situación. Por otro lado, la Universidad se prestaba a ser utilizada no fundamentalmente como un ámbito de docencia e investigación, sino como un mero ejercicio del poder académico o como una ayuda para saltar a otro ámbito de poder, sobre todo a la esfera política.

En resumen, todo lugar donde existan probabilidades de obtener poder o intercambiar influencias se convierte en un reducto fundamentalmente masculino, y aquí ese 29 por 100 de mujeres profesoras se reduce aún más. Por ello, son excluidas del poder universitario en el sentido que analizan Nieto (1984) y García de León $(1990)^{20}$.

La Universidad española actual mantiene una férrea disposición a no aceptar mujeres entre sus líderes. El mandarinato masculino sigue siendo el sistema de organización y poder universitario que se ha mantenido a lo largo de la última década ${ }^{21}$. Véanse, al respecto, los datos siguientes ${ }^{22}$ :

${ }^{20}$ Véase el trabajo sobre el poder universitario en la Universidad norteamericana de H. Adams, The academic tribes (Nueva York: Liberight, 1976). En España pueden mencionarse las contribuciones de A. NieTO, La tribu universitaria (Madrid: Tecnos, 1989), y María Antonia GARCÍA DE LEÓN, «Las profesoras universitarias: El caso de una élite discriminada», Revista Complutense de Educación, vol. 1, núm. 3 (1990).

${ }^{21}$ A. Almarcha, Autoridad y privilegio..., cit.; C. Fernández Villanueva, «La mujer en la universidad española: Docencia, investigación y poder. Datos y aspectos cualitativos», Revista de Educación, 290 (1989).

${ }^{22}$ Fuente: Instituto de la Mujer, 1989, y elaboración propia. En el curso 1992-93, la proporción de mujeres con cargos en la UCM era, en el caso de las Decanas, del 10,6 por 100; para las Secretarias Técnicas de Facultad la cifra era del 36,8 por 100, y, finalmente, las Directoras de Escuelas Universitarias representaban el 12,5 por 100. 


\begin{tabular}{|c|c|}
\hline & $\%$ mujeres \\
\hline - Rector & 0,2 \\
\hline 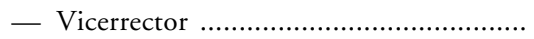 & 6,7 \\
\hline 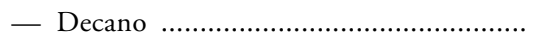 & 4,3 \\
\hline - Director de Escuela Universitaria ........... & 9,6 \\
\hline — Director de Departamento ........................ & 10,0 \\
\hline
\end{tabular}

\section{PARTICIPACION Y DISCRIMINACION EN LA UNIVERSIDAD COMPLUTENSE}

La situación de esta Universidad, una de las más masificadas y numerosas no sólo de España, sino teniendo en cuenta el ámbito europeo, no es menos preocupante (tabla 7). Veamos algunos aspectos relevantes: a) respecto a la categoría académica, las mujeres profesoras reducen su participación al 3,2 por 100 , es decir, por cada cinco varones catedráticos hay sólo una mujer; $b$ ) el techo para las mujeres sigue siendo el de ser Titular $(37,4)^{23} ; c$ ) las situaciones de privilegio que permiten las categorías de asociados, con sus diferentes tipos, también están reservadas a los varones $(28,8)$, frente a sólo un 16,3 de mujeres; d) el profesorado con menos expectativas, los ayudantes, constituye la categoría reservada a las mujeres $(18,7)$, frente a 5,7 de los varones.

A la hora de buscar una explicación que nos permita entender los desequilibrios existentes entre las distintas categorías de profesorado en función del sexo, encontramos que una explicación razonable de los mismos podría estar en la variable «edad». Efectivamente, las mujeres, que se han incorporado tardíamente al mundo educativo, son más jóvenes como media que los varones en el ámbito académico universitario. Un 33,4 por 100 de las docentes está en el grupo de las que tienen 35 años o menos, frente a sólo un 19,8 por 100 en el caso de los varones. De igual modo, un 27,4 por 100 de los profesores tiene más de 50 años, frente a sólo un 12,2 por 100 de las mujeres. ¿Podemos señalar que las docentes tienen aún que esperar para igualarse con los varones porque son demasiado jóvenes? Sin duda, una Universidad como la Complutense, con fama de ser un reducto para los más viejos, tiene, en la actualidad, un 42 por 100 de profesorado que, como máximo, tiene 40 años. Las mujeres, que, a menudo, acomodan su vida familiar, la maternidad y su carrera académica a la competitividad del puesto docente, si bien son algo más jóvenes que los varones, no parece que puedan obtener el mismo rendimiento que sus colegas.

${ }^{23}$ Datos del curso 1992/93 sitúan el porcentaje de catedráticos de Universidad en un 16,8 por 100 de varones y un 4,5 por 100 de mujeres. De igual modo, en las Escuelas Universitarias son catedráticos un 9,1 por 100 de varones, frente a un 4 por 100 de mujeres. Las mujeres titulares de Facultad alcanzaban el 50 por 100 y los varones el 36 por 100. Véase A. Almarcha y C. GonzÁlez Jorge, "La docencia en la Universidad Complutense de Madrid: Aspectos comparativos», en Seminario Internacional sobre Género y Trayectoria del Profesorado Universitario, Madrid, octubre 1993. 
Aunque el hecho de ser más jóvenes condiciona su situación dentro del mundo académico, no parece, sin embargo, que los méritos se establezcan en función de la edad o, expresado de otro modo, la edad sería una variable añadida a otros factores de desigualdad que explicaría una parte del fenómeno de la discriminación.

\section{TABLA 7}

Proporción de profesorado de Enseñanza Superior, según categoría académica y sexo, en la Universidad Complutense de Madrid (Curso 1990-1991)

\begin{tabular}{|c|c|c|c|}
\hline Categorias & Varones & Mujeres & Total \\
\hline Catedráticos y Agregados ...................... & 14,5 & 3,2 & 10,6 \\
\hline 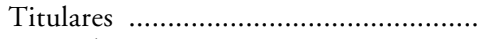 & 33,3 & 37,4 & 34,7 \\
\hline 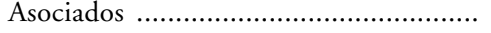 & 28,8 & 16,3 & 24,5 \\
\hline Ayudantes & 5,7 & 18,7 & 8,3 \\
\hline 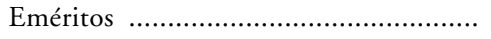 & 3,0 & 0,2 & 2,0 \\
\hline 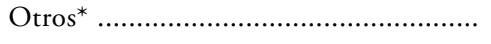 & 14,7 & 24,2 & 19,9 \\
\hline TОTAL & $\begin{array}{r}100,0 \\
(3.440)\end{array}$ & $\begin{array}{r}100,0 \\
(1.793)\end{array}$ & $\begin{array}{r}100,0 \\
(5.233)\end{array}$ \\
\hline
\end{tabular}

* Incluye Interinos y Profesores de Escuelas Universitarias dependientes de Departamentos de Facultad, es decir, en situaciones no consolidadas.

Fuente: Elaboración propia. Datos facilitados por la Sección de Personal de la UCM, diciembre 1990 (Curso 1990-1991). 


\title{
RESUMEN
}

El presente trabajo contiene parte de los resultados de una investigación más amplia realizada en la UCM sobre "la trayectoria profesional del profesorado de la Universidad Complutense», centrándose en los aspectos de género y carrera docente. El marco teórico se sitúa en la dualidad de roles y discriminación en el trabajo. Se pone, además, de relieve el cambio producido en las últimas décadas en cuanto a la participación de las mujeres en el sistema educativo, incidiendo preferentemente en la enseñanza superior.

\begin{abstract}
This paper is based on the results of a larger investigation carried out at the UCM. This research project deals with the "professional career of the university teachers of the Complutense University». The paper concentrates on the aspects of gender and teaching. The theoretical framework centers on the dual roles and discrimination of women at work. Emphasis is laid on the change that has taken place in the last decades regarding to the participation of women in the educational system, pointing up mainly problems found at he higher education level.
\end{abstract}

